\title{
ACUTE RESPONSES TO 4 VS. 4 SMALL-SIDED GAMES IN FOOTBALL PLAYERS
}

\author{
Pascual Bujalance-Moreno ${ }^{1}$, Pedro A. Latorre-Román ${ }^{1}$, Rodrigo Ramírez-Campillo ${ }^{2}$, \\ Antonio Martínez-Amat ${ }^{3}$, and Felipe García-Pinillos ${ }^{4}$ \\ ${ }^{1}$ Department of Corporal Expression, University of Jaen, Jaen, Spain \\ ${ }^{2}$ Department of Physical Activity Sciences, Research Nucleus in Health, \\ Physical Activity and Sport, Laboratory of Measurements and Assessment in Sport, \\ Universidad de Los Lagos, Osorno, Chile \\ ${ }^{3}$ Department of Health Sciences, University of Jaen, Jaen, Spain \\ ${ }^{4}$ Department of Physical Education, Sports and Recreation, \\ Universidad de La Frontera, Temuco, Chile
}

Original scientific article

DOI: $10.26582 / \mathrm{k} .52 .1 .7$

\begin{abstract}
:
The aim of this study was to examine acute physiological responses, physical fitness parameters and timemotion characteristics associated with a 4-a-side small-sided game in amateur level players. Sixteen male football players (age 23.9 \pm 4.2 years) completed one 4 -a-side small-sided game with the aim of maintaining ball possession as long as possible. The participants were monitored for external load and physiological parameters, and tested before and after for physical fitness assessment. A Student's paired $t$-test was conducted to determine the differences in physical fitness assessment. A repeated measures analysis of variance (ANOVA), with Bonferroni post-hoc test, was used to determine the dynamics of physiological parameters in players, their RPE and time-motion characteristics of SSG. A significant difference was found in $20 \mathrm{~m}$ sprint time between before and after the SSG $(\Delta=+1.3 \%)$. No significant differences were found between bouts or recovery periods for $\mathrm{THb}$ and $\mathrm{SmO}_{2}$, nor between HR-related variables (ES=.005-.383). Compared to RPE in bout 1 , greater values were observed in bouts 2,3 , and $4(\Delta=+5.3 \%, \mathrm{p}=.008, \mathrm{ES}=.40 ; \Delta=+9.6 \%$, $\mathrm{p}=.002, \mathrm{ES}=.98 ; \Delta=+15.1 \%, \mathrm{p}=.000, \mathrm{ES}=1.29$; respectively). No significant differences were found between bouts for time-motion characteristics. The results demonstrated that RPE responses increased throughout bout periods, whereas the rest of physiological parameters were maintained over the entire protocol. Due to possible fatigue accumulation, physical fitness performance was impaired (sprint) and the external load was reduced over the course of the protocol.
\end{abstract}

Key words: external/internal load, time-motion analysis, global positioning system, performance, rating of perceived exertion

\section{Introduction}

For a high match performance, it is essential to increase players' ability to maintain intense levels of activity and to limit fatigue at the same time, which means that players require a well-developed endurance (Stølen, Chamari, Castagna, \& Wisløff, 2005), as well as the ability of replicating physical fitness stimuli encountered in explosive football activities such as sprinting and jumping (Gehri, Ricard, Kleiner, \& Kirkendall, 1998) and physical responses (distance covered, speed, time) (Hill-Haas, Dawson, Coutts, \& Rowsell, 2009). In recent years, smallsided games (SSGs) have become a popular training drill for simultaneously improving fitness and tech- nical skills (Hill-Haas, Dawson, Impellizzeri, \& Coutts, 2011).

Through SSGs it is possible to improve physiological, physical fitness of players and their external load responses when certain variables are changed. Regarding intensity (i.e., physiological, physical fitness and external load responses) of SSGs, they may be modified according to the aims of training by changing several variables such as the number of players (Brandes, Heitmann, \& Müller, 2012), their roles (Sanchez-Sanchez, et al., 2017), dimensions of the playing area (Casamichana \& Castellano, 2010), among others. Given that each player has a specific physical potential, an indi- 
vidual assessment of player's physiological, physical fitness and external load responses is recommended for the optimization of training load application (Jastrzebski \& Radziminski, 2015). Furthermore, physiological responses to SSGs are skilland athletic-level-dependent (Dellal, et al., 2012). Therefore, data obtained from studies performed with professional players may not be applicable to amateur context, and vice versa.

With advancements in modern technology, individual and team performance can be simultaneously monitored to analyse internal (i.e., physiological and physical fitness) and external load (time-motion) responses. The global positioning system (GPS), heart-rate (HR) monitors, and rating of perceived exertion (RPE) are commonly used to quantify exercise intensity in football (Hill-Haas, et al., 2011). It has been suggested that SSGs training is best monitored via a combination of each of these measures of internal and external exercise intensity (using the new technologies that appear every day) (Coutts, Rampinini, Marcora, Castagna, \& Impellizzeri, 2009), facilitating simultaneous analyses of the training responses.

The analysis of physiological, physical fitness and external load responses is relatively common in the literature related to SSGs, especially to their 4-a-side protocols (Bujalance-Moreno, LatorreRomán, \& García-Pinillos, 2018; Hill-Haas, et al., 2011). However, to the best of the authors' knowledge, no previous studies have simultaneously examined the acute effects of a 4-a-side SSG on physiological (including muscle oxygen saturation $\left[\mathrm{SmO}_{2}\right]$ and total muscle haemoglobin [THb]), physical fitness, and time-motion profile of amateur football players. Therefore, the aim of this study was to examine the acute physiological responses, physical fitness parameters and time-motion characteristics associated with a 4-a-side SSG in amateurlevel players. It was hypothesized that the 4-a-side protocol would increase physiological demands to high levels, and simultaneously impair physical fitness and time-motion responses after a 4-a-side SSG.

\section{Methods}

\section{Procedures}

Athletes completed one 4-a-side SSG with the task of maintaining ball possession as long as possible, with no restrictions regarding the number of ball touches. The SSG protocol included four 4-minute bouts, played without goalkeepers on a pitch size of $30 \times 20 \mathrm{~m}$, with a 2-minute passive recovery between the bouts. When the ball was kicked out of play, a ball replacement was immediately provided. The SSG was supervised by one of the researchers (P.B.-M.). During recovery between the bouts, the RPE estimations (on a 6-20 scale) were obtained from the players by asking them: How hard was the SSG? (Borg, 1970).

\section{Participants}

Sixteen male football players (age 23.9 \pm 4.2 years) from two amateur teams with similar competitive and training schedules (eight players per team and each team played a SSG) successfully completed the study. Their standard training schedule involved three $2.6 \pm 0.5$ hours training sessions per week and a weekly league match. The study was conducted during their competition season. Participants had been involved in regular football training for at least two years prior to the study. Measurements were performed as part of their regular training and testing programme, and players approved the use of these data for research purposes. Moreover, players and coaches were fully informed about the potential risks and benefits derived from participation in the study protocol, and signed an informed consent document before participating in the investigation. The study was conducted in adherence to the standards of the Declaration of Helsinki (2013 version). The local ethics committee approved the informed consent and the study design.

\section{Physical fitness assessment}

Sprint evaluation (pre- and post-SSG) was accomplished through a speed test that was carried out in a straight $20 \mathrm{~m}$ line (Maio Alves, Rebelo, Abrantes, \& Sampaio, 2010). Sprint times (in seconds) were measured using two double-light barriers (Witty; Microgate Srl, Bolzano, Italy; accuracy of 0.001 second). The players performed two trials with a 3-minute recovery in-between. A better result was recorded for further analysis. The countermovement vertical jump (CMJ) (pre- and postSSG) was assessed through a vertical jump test. The participants were highly familiarized with the CMJ technique (Ramirez-Campillo, Andrade, \& Izquierdo, 2013), as they performed CMJ in their training sessions. The CMJ was recorded using the OptoGait system (Microgate, Bolzano, Italy), which was also used in a similar study (Lehance, Binet, Bury, \& Croisier, 2009). Subjects performed three trials with a 15 -second recovery period between them, and the best trial was used for the statistical analysis.

\section{Physiological characteristics and RPE estimations}

Session RPE was recorded, on an individual basis, immediately after each of the four bouts of the SSG, using the 6-20 scale (Borg, 1970). Familiarization with the RPE scale was completed before the SSG. Players' HR was recorded at 5-second intervals during the 4-a-side SSG via short-range radio telemetry (Polar Team Sport System, Polar Electro Oy, Finland). It was expressed as the 
percentage of peak HR (HRpeak) as measured using generalized equation for predicting HRpeak (Tanaka, Monahan, \& Seals, 2001) and classified into four previously defined intensity zones: Zone 1 (<75\% HRpeak), Zone 2 (75-84\% HRpeak), Zone 3 (85-89\% HRpeak), and Zone 4 ( $\geq 90 \%$ HRpeak) (Casamichana \& Castellano, 2010). The percentage of time spent within each intensity zone during the SSG was quantified, as well as the percentages of HRpeak (\%HRpeak), and average HR (HRaverage) with respect to the calculated one through the generalized equation proposed by Tanaka et al. (2001). In addition, the HRpeak in the task was recorded during the work and recovery periods of the SSG. $\mathrm{The} \mathrm{SmO}_{2}$ and $\mathrm{THb}$ was monitored by near infrared spectroscopy (Moxy, Fortiori Design LLC, Minnesota, USA), which was also used in a similar study (Crum, O'Connor, Van Loo, Valckx, \& Stannard, 2017). The Moxy was positioned on the participant's dominant leg, as in a previous study (Crum, et al., 2017). Leg dominance was determined by asking the athletes about their preferred kicking leg. The Moxy is a reliable device to measure $\mathrm{SmO}_{2}$ and $\mathrm{THb}$, validated in a previous study (Crum, et al., 2017). To know when variables were measured see Figure 1.

\section{Time-motion characteristics}

Time-motion characteristics of playing the SSG were measured using Ultra Wide Band-based (UWB) position-tracking systems units (WIMU $\mathrm{PRO}^{\mathrm{TM}}$ ) and analysed using $\mathrm{S} \mathrm{PRO}^{\mathrm{TM}}$ software (RealTrack Systems, Almeria, Spain). The UWB system was positioned as in a previous study (Bastida Castillo, Gómez Carmona, De la Cruz Sánchez, \& Pino Ortega, 2018). The reliability and validity of this technology for monitoring players' high-intensity and sprinting activities during football matches has been previously determined (Bastida Castillo, et al., 2018). For data analysis purposes, four speed zones were selected: speed zone 1 (standing and walking, 0.1-6.9 km/h), speed zone 2 (low-intensity running, $7.0-12.9 \mathrm{~km} / \mathrm{h}$ ), speed zone 3 (medium-intensity running, 13.0-17.9 km/h), and speed zone 4 (high-intensity running, $\geq 18.0$ $\mathrm{km} / \mathrm{h}$ ) (Casamichana, Suarez-Arrones, Castellano, \& San Román-Quintana, 2014; Hill-Haas, et al., 2009); and four acceleration zones: acceleration zone $1\left(1.0-1.4 \mathrm{~m} / \mathrm{s}^{2}\right)$, acceleration zone $2(1.5-$ $\left.1.9 \mathrm{~m} / \mathrm{s}^{2}\right)$, acceleration zone $3\left(2.0-2.4 \mathrm{~m} / \mathrm{s}^{2}\right)$, and acceleration zone $4\left(\geq 2.5 \mathrm{~m} / \mathrm{s}^{2}\right)$ (Casamichana, et al., 2014). Similarly to previous studies, sprint distance was established ( $>24.1 \mathrm{~km} / \mathrm{h})$ (Dellal, et al., 2012). The total distance travelled, total $\mathrm{m} / \mathrm{min}$, total accelerations and decelerations, sprints, average sprint duration, maximal speed reached, average speed and distance travelled within the designated speed zones and the number of accelerations within the designated zones were calculated.

\section{Experimental protocols}

This is a cross-sectional study in which participants were monitored for external load and physiological parameters during a 4-a-side SSG protocol, and tested before and after (pre- and post-SSG) for physical fitness assessment (see Figure 1). The participants were asked to avoid high-intensity exercise $\geq 72$ hours before the testing sessions, and to be well hydrated and have a carbohydrate-rich meal $\geq 2$ hours before the beginning of the testing protocols. The tests were completed on the out-door artificial grass surface, where athletes usually trained and competed. Before testing, athletes completed a typical warm-up consisting of a 5-minute low intensity running and a 5-minute high skipping, leg lifts, lateral running, and sprints. The participants were motivated to perform at maximal intensity in every test. The athletes were familiarized with the SSG protocol, as they use it as part of their regular training sessions. The players were randomly assigned to one of the two teams.

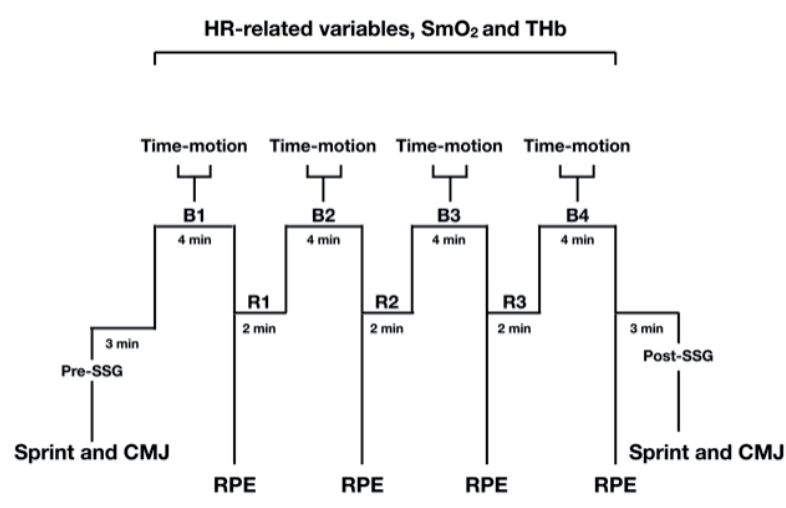

Figure 1. Schematic of the experimental protocol and variable measurements.

\section{Statistical analyses}

Descriptive statistics are represented as mean (M) and standard deviation (SD). Tests of distribution normality and homogeneity (Shapiro-Wilk and Levene's test, respectively) were conducted on all data before further analysis. A Student's paired $t$-test was conducted to determine the pre- vs. post-SSG differences in physical fitness assessment (CMJ and sprint test). A repeated measures analysis of variance (ANOVA), with the Bonferroni post-hoc test, was used to determine the dynamics of physiological parameters, RPE and time-motion characteristics during the SSG protocol. Changes $(\Delta)$ between the four bouts of the SSG was calculated in percentage (\%). The magnitude of the differences between values was also interpreted using the Cohen's d effect size $\left({ }^{\mathrm{ES}}\right)$ (Thomas, Silverman, \& Nelson, 2015). Effect sizes of $<.4$ represented a small magnitude of change, whereas $.41-.7$ and $>.7$ represented moderate and large magnitudes of change, respectively (Thomas, 
et al., 2015). Finally, a Pearson correlation analysis was conducted between the changes induced by the SSG protocol in all variables analysed $(\Delta$ : bout 4 - bout 1$)$. The following criteria were adopted to interpret the magnitude of correlations between measurement variables: <.1 (trivial), .1-.3 (small), .3-.5 (moderate), .5-.7 (large), .7-.9 (very large) and .9-1.0 (almost perfect) (Hopkins, Marshall, Batterham, \& Hanin, 2009). Data analysis was performed using SPSS (version 22, SPSS Inc., Chicago, IL, USA), and the significance level was set at $\mathrm{p}<.05$.

\section{Results}

\section{Physical fitness assessment}

Table 1 shows the results of physical fitness assessments (sprint and jump) before and after the 4-a-side SSG. A significant difference was found in
$20 \mathrm{~m}$ sprint between times before and after the SSG $(\Delta=+1.3 \%)$. No significant differences in $5 \mathrm{~m}$ sprint $(\Delta=+.9 \%)$, or $\mathrm{CMJ}(\Delta=-.6 \%)$ were found between values before and after the SSG.

\section{Physiological characteristics and RPE estimations}

Table 2 shows the physiological characteristics of players during the SSG and their RPE estimations. No significant differences were found between bouts or recovery periods for $\mathrm{THb}$ and $\mathrm{SmO}_{2}$ nor for HR-related variables ( $\mathrm{ES}=.005-.383$ ). Compared to RPE in bout 1 , greater values were observed in bouts 2,3 , and $4(\Delta=+5.3 \%, \mathrm{p}=.008, \mathrm{ES}=.40$; $\Delta=+9.6 \%, \mathrm{p}=.002, \mathrm{ES}=.98 ; \Delta=+15.1 \%, \mathrm{p}=.000$, $\mathrm{ES}=1.29$; respectively). Moreover, compared to RPE in bout 2 and 3 , greater values were observed in bout $4(\Delta=+10.3 \%, \mathrm{p}=.004, \mathrm{ES}=.78 ; \Delta=+6.1 \%$, $\mathrm{p}=.049, \mathrm{ES}=.55$; respectively).

Table 1. Physical fitness assessment before and after a 4-a-side small-sided game in amateur football players $(n=16)$

\begin{tabular}{|c|c|c|c|c|}
\hline & Before & After & $p$-value & Effect size \\
\hline 20-m sprint (s) & $3.11 \pm .14$ & $3.15 \pm .12$ & $p=.02$ & .306 \\
\hline 5-m sprint (s) & $1.08 \pm .08$ & $1.09 \pm .05$ & $p=.42$ & .149 \\
\hline Countermovement jump (cm) & $39.1 \pm 5.5$ & $38.9 \pm 5.8$ & $p=.67$ & .040 \\
\hline
\end{tabular}

Table 2. Physiological and perceptual responses associated with a 4-a-side small-sided game in amateur football players ( $n=16)$

\begin{tabular}{|c|c|c|c|c|}
\hline & Bout* 1 & Bout 2 & Bout 3 & Bout 4 \\
\hline Total haemoglobin content $\left(\mathrm{g} \cdot \mathrm{dL}^{-1}\right)$ & $12.1 \pm .5$ & $12.2 \pm .5$ & $12.1 \pm .5$ & $12.1 \pm .5$ \\
\hline Muscle oxygen saturation (\%) & $36.2 \pm 15.3$ & $38.4 \pm 17.4$ & $34.0 \pm 13.2$ & $34.3 \pm 15.3$ \\
\hline Rating of perceived exertion (6-20) & $12.3 \pm 1.5$ & $13.0 \pm 1.9^{a}$ & $13.6 \pm 1.2^{\mathrm{a}}$ & $14.5 \pm 1.9^{a, b, c}$ \\
\hline $\mathrm{HR}_{\text {peak }}\left(\right.$ beats $\left.\cdot \mathrm{min}^{-1}\right)$ & $169 \pm 23.9$ & $174 \pm 17.6$ & $171 \pm 21.0$ & $172 \pm 18.7$ \\
\hline $\mathrm{HR}_{\text {average }}\left(\right.$ beats $\cdot \mathrm{min}^{-1}$ ) & $157 \pm 23.9$ & $161 \pm 19.4$ & $160 \pm 18.6$ & $159 \pm 20.2$ \\
\hline$\% \operatorname{HR}_{\text {peak }}(\%)$ & $87.0 \pm 10.5$ & $88.9 \pm 8.3$ & $88.5 \pm 6.9$ & $88.3 \pm 8.6$ \\
\hline$<75 \% \mathrm{HR}_{\text {peak }}(\%)$ & $25.6 \pm 34.5$ & $21.3 \pm 30.6$ & $19.6 \pm 32.6$ & $20.5 \pm 34.1$ \\
\hline $75-84 \% \mathrm{HR}_{\text {peak }}(\%)$ & $10.3 \pm 8.7$ & $14.5 \pm 10.4$ & $13.7 \pm 15.3$ & $11.6 \pm 11.0$ \\
\hline $85-89 \% \mathrm{HR}_{\text {peak }}(\%)$ & $7.7 \pm 5.8$ & $7.5 \pm 7.8$ & $10.7 \pm 13.6$ & $10.8 \pm 10.0$ \\
\hline \multirow[t]{2}{*}{$>90 \% \mathrm{HR}_{\text {peak }}(\%)$} & $56.4 \pm 30.5$ & $56.8 \pm 32.0$ & $56.0 \pm 31.4$ & $57.0 \pm 32.2$ \\
\hline & $\begin{array}{l}\text { Recovery between } \\
\text { bouts } 1 \text { and } 2\end{array}$ & \multicolumn{2}{|c|}{$\begin{array}{l}\text { Recovery between } \\
\text { bouts } 2 \text { and } 3\end{array}$} & $\begin{array}{l}\text { Recovery between bouts } \\
\qquad 3 \text { and } 4\end{array}$ \\
\hline Total haemoglobin content $\left(\mathrm{g} \cdot \mathrm{dL}^{-1}\right)$ & $12.4 \pm .4$ & \multicolumn{2}{|c|}{$12.2 \pm .5$} & $12.4 \pm .3$ \\
\hline Muscle oxygen saturation (\%) & $66.7 \pm 8.7$ & \multicolumn{2}{|c|}{$65.2 \pm 9.9$} & $61.1 \pm 15.4$ \\
\hline $\mathrm{HR}_{\text {peak }}\left(\right.$ beats $\cdot \mathrm{min}^{-1}$ ) & $167 \pm 24.5$ & \multicolumn{2}{|c|}{$169 \pm 20.1$} & $165.6 \pm 23.1$ \\
\hline $\mathrm{HR}_{\text {average }}\left(\right.$ beats $\left.\cdot \mathrm{min}^{-1}\right)$ & $143 \pm 17.7$ & \multicolumn{2}{|c|}{$144 \pm 11.3$} & $145 \pm 16.9$ \\
\hline$\% \mathrm{HR}_{\text {peak }}(\%)$ & $79.2 \pm 8.8$ & \multicolumn{2}{|c|}{$79.8 \pm 3.4$} & $80.1 \pm 6.7$ \\
\hline$<75 \% \mathrm{HR}_{\text {peak }}(\%)$ & $42.1 \pm 34.0$ & \multicolumn{2}{|c|}{$36.8 \pm 33.5$} & $41.5 \pm 38.9$ \\
\hline $75-84 \% \mathrm{HR}_{\text {peak }}(\%)$ & $29.2 \pm 25.2$ & \multicolumn{2}{|c|}{$26.2 \pm 18.0$} & $22.2 \pm 17.6$ \\
\hline $85-89 \% \mathrm{HR}_{\text {peak }}(\%)$ & $9.1 \pm 5.8$ & \multicolumn{2}{|c|}{$11.9 \pm 9.3$} & $13.6 \pm 18.9$ \\
\hline$>90 \% \mathrm{HR}_{\text {peak }}(\%)$ & $19.6 \pm 16.6$ & \multicolumn{2}{|c|}{$18.9 \pm 13.1$} & $22.7 \pm 25.7$ \\
\hline
\end{tabular}

Note. $\mathrm{HR}_{\text {peak }}=$ peak heart rate; $\mathrm{HR}_{\text {average }}=$ average heart rate; $\% \mathrm{HR}_{\text {peak }}=$ percentage of $\mathrm{HR}_{\text {peak }} ;<75 \% \mathrm{HR}_{\text {peak }}(\%)=$ the percentage of time spent within that intensity zone; $75-84 \% \mathrm{HR}_{\text {peak }}(\%)=$ the percentage of time spent within that intensity zone; $85-89 \% \mathrm{HR}_{\text {peak }}(\%)=$ the percentage of time spent within that intensity zone; $>90 \% \mathrm{HR}_{\text {peak }}(\%)=$ the percentage of time spent within that intensity zone. ${ }^{\mathrm{a}, \mathrm{b}, \mathrm{c}}$ : different $(p<.05)$ compared to bout 1 , bout 2 , and bout 3 , respectively. *: each bout lasted 4 minutes. 
Table 3. Time-motion characteristics associated with a 4-a-side small-sided game in amateur football players $(n=16)$

\begin{tabular}{lcccc}
\hline & Bout* 1 & Bout 2 & Bout 3 & Bout 4 \\
\hline Total distances $(\mathrm{m})$ & $533 \pm 48.3$ & $515 \pm 59.5$ & $500 \pm 53.4^{\mathrm{a}}$ & $512 \pm 42.9$ \\
Total distances at $0.1-6.9 \mathrm{~km} \cdot \mathrm{h}^{-1}(\mathrm{~m})$ & $179 \pm 20.9$ & $183 \pm 23.7$ & $200 \pm 28.0^{\mathrm{a}}$ & $193 \pm 17.8$ \\
Total distances at $7.0-12.9 \mathrm{~km} \cdot \mathrm{h}^{-1}(\mathrm{~m})$ & $289 \pm 45.2$ & $258 \pm 49.3$ & $249 \pm 52.1^{\mathrm{a}}$ & $256 \pm 46.7^{\mathrm{a}}$ \\
Total distances at $13.0-17.9 \mathrm{~km} \cdot \mathrm{h}^{-1}(\mathrm{~m})$ & $62.1 \pm 18.9$ & $67.5 \pm 33.3$ & $49.9 \pm 27.5$ & $58.1 \pm 13.3$ \\
Total distances at $\geq 18.0 \mathrm{~km} \cdot \mathrm{h}^{-1}(\mathrm{~m})$ & $1.8 \pm 3.0$ & $6.5 \pm 6.6$ & $2.0 \pm 2.3$ & $6.1 \pm 8.2$ \\
Total $\mathrm{m} /$ min & $133 \pm 11.9$ & $128 \pm 14.7$ & $125 \pm 13.4^{\mathrm{a}}$ & $128 \pm 10.9$ \\
Total number of accelerations & $231 \pm 10.7$ & $232 \pm 19.3$ & $233 \pm 13.7$ & $233 \pm 12.2$ \\
Number of accelerations between $1.0-1.4 \mathrm{~m} \cdot \mathrm{s}^{-2}$ & $36.3 \pm 8.6$ & $42.8 \pm 11.2$ & $43.1 \pm 8.1^{\mathrm{a}}$ & $46.8 \pm 4.6^{\mathrm{a}}$ \\
Number of accelerations between $1.5-1.9 \mathrm{~m} \cdot \mathrm{s}^{-2}$ & $30.8 \pm 9.2$ & $36.9 \pm 7.9$ & $36.3 \pm 8.8$ & $39.1 \pm 6.4^{\mathrm{a}}$ \\
Number of accelerations between $2.0-2.4 \mathrm{~m} \cdot \mathrm{s}^{-2}$ & $25.7 \pm 6.4$ & $27.0 \pm 5.6$ & $28.1 \pm 6.0$ & $28.5 \pm 5.3$ \\
Number of accelerations $\geq 2.5 \mathrm{~m} \cdot \mathrm{s}^{-2}$ & $138 \pm 19.1$ & $125 \pm 20.5$ & $126 \pm 13.3$ & $119 \pm 9.6^{\mathrm{a}}$ \\
Total number of decelerations & $231 \pm 12.2$ & $229 \pm 10.3$ & $228 \pm 14.3$ & $232 \pm 15.2$ \\
Average sprint duration $(\mathrm{s})$ & - & - & - & $.03 \pm .1$ \\
Total number of sprints & - & - & - & $.06 \pm .3$ \\
Maximal speed $\left(\mathrm{km} \cdot \mathrm{h}^{-1}\right)$ & $18.0 \pm 1.5$ & $19.5 \pm 2.2$ & $18.2 \pm 1.8$ & $19.9 \pm 2.7^{\mathrm{a}}$ \\
Average speed $\left(\mathrm{km} \cdot \mathrm{h} \mathrm{h}^{-1}\right)$ & $7.0 \pm .4$ & $6.8 \pm .8$ & $6.5 \pm .6^{\mathrm{a}}$ & $6.7 \pm .5^{\mathrm{a}}$ \\
\hline
\end{tabular}

Note. a: denotes differences $(p<.05)$ compared to values in bout 1. *: each bout lasted 4 minutes.

\section{Time-motion characteristics}

The time-motion characteristics obtained for each of the four bouts of the SSG are indicated in Table 3. A shorter distance travelled at 0.1-6.9 $\mathrm{km} / \mathrm{h}$ was observed in bout 1 versus bout 3 ( $\mathrm{p}=.036$, $\mathrm{ES}=.83$ ). A longer distance travelled at 7.0-12.9 $\mathrm{km} / \mathrm{h}$ was observed in bout 1 versus bout 3 ( $\mathrm{p}=.005$, $\mathrm{ES}=.82)$ and bout $4(\mathrm{p}=.007, \mathrm{ES}=.71)$. A significant time-effect was found in distance travelled at $\geq 18.0$ $\mathrm{km} / \mathrm{h}(\mathrm{p}=.049, \mathrm{ES}=.05-.91)$, but post-hoc analysis reported no significant differences between the four bouts of the SSG. Regarding total distance travelled and $\mathrm{m} / \mathrm{min}$, greater values were observed in bout 1 versus 3 ( $\mathrm{p}=.021, \mathrm{ES}=.62$ and $\mathrm{p}=.019, \mathrm{ES}=.61$; respectively). Maximal speed was greater in bout 4 versus bout $1(\mathrm{p}=.005, \mathrm{ES}=.85)$, while average speed was greater in bout 1 versus bouts 3 and 4 ( $\mathrm{p}=.012, \mathrm{ES}=.94$; and $\mathrm{p}=.039, \mathrm{ES}=.79$; respectively). The number of accelerations between 1.0-1.4 m.s. was bigger in bout 3 and 4 versus bout $1(\mathrm{p}=.038$, $\mathrm{ES}=.81$; and $\mathrm{p}=.008, \mathrm{ES}=1.51$; respectively). The number of accelerations between 1.5-1.9 m.s. $\mathrm{s}^{-2}$ was bigger in bout 4 versus bout $1(\mathrm{p}=.031, \mathrm{ES}=1.05)$, while the number of accelerations $\geq 2.5 \mathrm{~m} \cdot \mathrm{s}^{-2}$ was bigger in bout 1 versus bout $4(\mathrm{p}=.015, \mathrm{ES}=1.27)$. No other differences were found between the bouts.

Finally, the Pearson correlation analysis revealed some significant correlations between the changes induced by the SSG protocol in the analysed variables. Changes in sprinting and jumping performance significantly correlated $(\mathrm{r}=.810, \mathrm{p}<.01)$. Significant correlations were also found between $\triangle \mathrm{RPE}$ with $\Delta$ HRpeak and $\Delta$ HRaverage $(.5<\mathrm{r}>.6, \mathrm{p}<.05)$, and with $\Delta<75 \%$ HRpeak and $\Delta \geq 90 \%$ HRpeak ( $\mathrm{r}=-.521$ and .619 , respectively, $\mathrm{p}<.05)$. Significant correla- tions $(\mathrm{p}<.05)$ were found between time-motion parameters (i.e., $\Delta$ total distance correlated with $\Delta \mathrm{m} / \mathrm{min}$ and $\Delta$ average speed, $\mathrm{r}>.8$ in both cases).

\section{Discussion and conclusions}

This study aimed to examine acute physiological and physical fitness responses to as well as time-motion characteristics associated with a 4-a-side SSG in amateur football players. The main findings indicated that sprint performance was impaired after the SSG, whereas jumping performance remained unchanged. Moreover, the RPE increased throughout the four bouts of the SSG, although without changes in $\mathrm{HR}, \mathrm{THb}$ and $\mathrm{SmO}_{2}$. In addition, during the recovery periods, no changes were observed in $\mathrm{HR}, \mathrm{THb}$ and $\mathrm{SmO}_{2}$. Regarding time-motion characteristics, some changes were observed in the last bouts of the protocol compared to the first one.

\section{Physical fitness performance}

The effect of SSGs on sprint and jumping capacities has received the attention of some studies (Bujalance-Moreno, García-Pinillos, \& LatorreRomán, 2017; Clemente, Nikolaidis, Van Der Linden, \& Silva, 2017). The results of the current study revealed an impairment in $20 \mathrm{~m}$ sprint performance after the SSG protocol. These results are consistent with previous studies examining 3-a-side and 6-a-side SSGs (Katis \& Kellis, 2009). Sprint running involves stretch-shortening cycle muscle function of both the lower and upper body musculature with increased metabolic demands (Delecluse, 1997). As indicated by a previous study (Katis \& 
Kellis, 2009), it seems that higher endurance levels are needed to maintain athletic performance. This might help to explain the reduction in sprinting performance after the 4-a-side SSG. In contrast to sprinting, the CMJ is characterised by a short and quick production of force and power, especially by the knee extensors. The maintenance of jump scores following our protocol agrees with previous findings which showed the same values of jump performance after the SSG protocol (Clemente, et al., 2017). The type of muscular contraction and recruitment might apparently explain the fact that both running and jumping were relatively independent (Buchheit, 2010). In addition, Dellal, Hill-Haas, Lago-Penas, and Chamari (2011) explained that the 4-a-side SSG induced less fatigue in jumping capabilities compared to the 2-a-side SSG protocol due to a relative decrease in high-intensity actions and sprinting in the proportion to the total distance covered in the former SSG protocol versus the latter. These results may increase the knowledge about the effect of the 4-a-side SSG on sprint and jumping capacities in amateur football players. Using the results of this study, coaches may be able to predict some players' responses in the specific training context, so this can help coaches to plan better training sessions.

\section{Physiological characteristics and RPE estimations}

Rate of Perceived Exertion (RPE) has been suggested to be a good indicator of exercise intensity when compared with HR or blood lactate during football-specific exercise (Hill-Haas, et al., 2011). In the present study, the RPE increased throughout the entire protocol, which seems logical considering high physical demands (i.e., in terms of external load) and the accumulation of fatigue. This can also be attributed to the exercise intensity that was increasing during the protocol, as shown by the correlation analysis between $\triangle \mathrm{RPE}$ and $\Delta$ HR-related variables. Compared to previous studies that used this SSG format, higher (HillHaas, et al., 2009), similar (Dellal, Lago-Penas, Wong, \& Chamari, 2011) and lower (Brandes \& Elvers, 2016) RPE values were reported. In addition to the SSG format, the intensity of the game, and thereby the RPE, depends on several variables such as the number of players (Brandes, et al., 2012; Hill-Haas, et al., 2009), or dimensions of the playing area (Casamichana \& Castellano, 2010), and that might explain the lack of consistency with previous studies.

Monitoring training sessions, especially the HR response, has been suggested as a useful method to regulate exercise intensity (Hill-Haas, et al., 2011). It seems clear that SSG formats with fewer players elicit greater \%HR (Hill-Haas, et al., 2009). Likewise, SSGs played on different pitch sizes had a similar intensity (in terms of HR response)
(Hodgson, Akenhead, \& Thomas, 2014). In the present study, the 4-a-side SSG format elicited a smaller amount of time spent at $>90 \%$ HRpeak $(\Delta=-2.69 \%)$, which can be due to a shorter duration of SSG and recovery between bouts, and a higher \%HRpeak response $(\Delta=+3.57 \%)$ than the 4-a-side format of previous researches (Hill-Haas, et al., 2009). Altogether, these results demonstrate a moderate correlation between HR and RPE responses. The current study suggest that this SSG format (4-a-side without goalkeepers and only ball possession maintenance) may be an useful tool for improving specific endurance in football players (Brandes, et al., 2012).

In terms of muscle oxygenation characteristics $\left(\mathrm{SmO}_{2}, \mathrm{THb}\right)$, the available evidence is limited. A previous study pointed to muscle oxygenation characteristics as an acceptable index of metabolic demand in the working muscle (Crum, et al., 2017). In the aforementioned study (Crum, et al., 2017), when exercise intensity increased, a decrease in $\mathrm{SmO}_{2}$ was reported. In contrast, $\mathrm{THb}$ showed little variation throughout each trial and was not associated with changes in exercise intensity. On the other hand, in the current study, $\mathrm{SmO}_{2}$ remained unchanged throughout four bouts. Moreover, during the recovery periods between the four bouts of the SSG, the $\mathrm{SmO}_{2}$ values remained unchanged $(\sim 74-78 \%)$ over the entire protocol. As for $\mathrm{THb}$, the current results are consistent with those of a previous study that observed no changes over an incremental cycling protocol (Crum, et al., 2017). Since $\mathrm{SmO}_{2}$ measurements have showed a negative correlation with oxygen uptake and HR (Crum, et al., 2017), the results reported by the current study seems logical with HR being constant during both work and rest periods and, thereby, constant $\mathrm{SmO}_{2}$ values. The current study reported no significant correlations between $\Delta \mathrm{HR}$-related variables and $\Delta$ Moxy-related variables. The authors suggest that the lack of baseline values for these parameters (pre-SSG data, at rest) makes differences between work periods or between recovery periods too small (very stable over time), which makes difficult finding significant correlations. Anyway, future studies are required to increase the understanding of muscle oxygenation characteristics during football-specific tasks.

\section{Time-motion characteristics}

Previous studies examined the effects of SSG formats on the external load (Jastrzebski \& Radziminski, 2015). In general, modifications in the number of players and the balance of the opposition have been shown to alter the total distance covered (Hill-Haas, et al., 2009) as well as high-intensity running and sprinting performances (Hill-Haas, et al., 2009; Jastrzebski \& Radziminski, 2015). HillHaas et al. (2009) found that the 4-a-side format 
was accompanied by greater physical demands compared to those observed in the current work, although in the current study a smaller pitch size was used. These results suggest that the pitch size is a very important variable that influences the player's workload, especially high intensity actions (Casamichana, Castellano, \& Castagna, 2012; Dellal, et al., 2012). However, compared with our results, a previous work (Dellal, Lago-Penas, et al., 2011) showed a greater total distance travelled and total distance travelled in sprinting in a 4-a-side SSG with free play and the same pitch size. Additionally, other study (Hodgson, Akenhead, \& Thomas, 2014) obtained a smaller amount of physical demands (i.e., external load) than our study, using equal pitch size, but including goalkeepers. In this study, no changes were found throughout the bouts on external load (i.e., total distance, number of accelerations), but the intensity of game was reduced. Thus, the highest total distance travelled was obtained in bout 1 , which was in contradiction with the highest internal load (RPE) observed in the last bout. This can indicate that the increment in RPE from bout 2, despite the decreased external load, may be due to fatigue accumulation across the bouts. Those findings are similar to those found in previous studies (Clemente, et al., 2017). Nevertheless, caution is advised when interpreting current findings, owing to the heterogeneity that exists among study protocols.
The knowledge about the effects caused by each manipulation of variables seems to play a key role.

Some limitations of the current study must be addressed. First, the sample size was relatively small, which might limit the generalization of findings. Second, the lack of data about the players' technical performance, which might add useful information. Notwithstanding those limitations, the current work provides some insights into the demands of a typical SSG format (i.e., 4-a-side SSG).

In conclusion, this 4-a-side SSG format implies that RPE responses increased throughout bout periods, whereas the rest of physiological parameters $\left(\mathrm{SmO}_{2}, \mathrm{THb}\right.$ and HR-related variables) were maintained over the entire protocol. Due to possible fatigue accumulation and, therefore, to the decrease of game intensity, the physical fitness performance was impaired (sprint) and the external load was reduced over the course of the protocol. From a practical standpoint, the results obtained provide some insights into the acute impact of a widely used SSG format. Coaches and technical stuff might find this information very useful for expanding their knowledge about how this protocol influences football players from a multidisciplinary (physical fitness, physiological and time-motion) approach.

\section{References}

Bastida Castillo, A., Gómez Carmona, C.D., De la Cruz Sánchez, E., \& Pino Ortega, J. (2018). Accuracy, intra- and interunit reliability, and comparison between GPS and UWB-based position-tracking systems used for time-motion analyses in soccer. European Journal of Sport Science, 18(4), 450-457. doi: 10.1080/17461391.2018.1427796

Borg, G. (1970). Perceived exertion as an indicator of somatic stress. Scandinavian Journal of Rehabilitation and Medicine, 2, 92-98.

Brandes, M., \& Elvers, S. (2016). Elite youth soccer players' physiological responses, time-motion characteristics, and game performance in 4 vs. 4 small-sided games: The influence of coach feedback. Journal of Strength and Conditioning Research, 31(10), 2652-2658.

Brandes, M., Heitmann, A., \& Müller, L. (2012). Physical responses of different small-sided game formats in elite youth soccer players. Journal of Strength and Conditioning Research, 26(5), 1353-1360.

Buchheit, M. (2010). Performance and physiological responses to repeated-sprint and jump sequences. European Journal of Applied Physiology, 110(5), 1007-1018.

Bujalance-Moreno, P., García-Pinillos, F., \& Latorre-Román, P.A. (2017). Effects of a small-sided game-based training programme on repeated sprint and change of direction abilities in recreationally-trained football players. The Journal of Sports Medicine and Physical Fitness, 58(7-8), 1021-1028.

Bujalance-Moreno, P., Latorre-Román, P.Á., \& García-Pinillos, F. (2018). A systematic review on small-sided games in football players: Acute and chronic adaptations. Journal of Sports Sciences, 37(8), 921-949.

Casamichana, D., \& Castellano, J. (2010). Time-motion, heart rate, perceptual and motor behaviour demands in smallsides soccer games: Effects of pitch size. Journal of Sports Sciences, 28(14), 1615-1623.

Casamichana, D., Castellano, J., \& Castagna, C. (2012). Comparing the physical demands of friendly matches and smallsided games in semiprofessional soccer players. Journal of Strength and Conditioning Research, 26(3), 837-843.

Casamichana, D., Suarez-Arrones, L., Castellano, J., \& San Román-Quintana, J. (2014). Effect of number of touches and exercise duration on the kinematic profile and heart rate response during small-sided games in soccer. Journal of Human Kinetics, 41, 113-123. 
Clemente, F.M., Nikolaidis, P.T., Van Der Linden, C.M.I.N., \& Silva, B. (2017). Effects of small-sided soccer games on internal and external load and lower limb power: A pilot study in collegiate players. Human Movement, $18(1), 50-57$.

Coutts, A.J., Rampinini, E., Marcora, S.M., Castagna, C., \& Impellizzeri, F.M. (2009). Heart rate and blood lactate correlates of perceived exertion during small-sided soccer games. Journal of Science and Medicine in Sport, 12(1), 79-84.

Crum, E.M., O’Connor, W.J., Van Loo, L., Valckx, M., \& Stannard, S.R. (2017). Validity and reliability of the Moxy oxygen monitor during incremental cycling exercise. European Journal of Sport Science, 17(8), 1037-1043.

Delecluse, C. (1997). Influence of strength training on sprint running performance. Sports Medicine, 24(3), 147-156.

Dellal, A., Hill-Haas, S., Lago-Penas, C., \& Chamari, K. (2011). Small-sided games in soccer: Amateur vs. professional players' physiological responses, physical, and technical activities. Journal of Strength and Conditioning Research, 25(9), 2371-2381.

Dellal, A., Lago-Penas, C., Wong, D., \& Chamari, K. (2011). Effect of the number of ball contacts within bouts of 4 vs. 4 small-sided soccer games. International Journal of Sports Physiology and Performance, 6(3), 322-333.

Dellal, A., Owen, A., Wong, D., Krustrup, P., Van Exsel, M., \& Mallo, J. (2012). Technical and physical demands of small vs. large sided games in relation to playing position in elite soccer. Human Movement Science, 31, 957-969.

Gehri, D.J., Ricard, M.D., Kleiner, D.M., \& Kirkendall, D.T. (1998). A comparison of plyometric training techniques for improving vertical jump ability and energy production. Journal of Strength and Conditioning Research, 12(2), 85-89.

Hill-Haas, S.V., Dawson, B.T., Coutts, A.J., \& Rowsell, G.J. (2009). Physiological responses and time-motion characteristics of various small-sided soccer games in youth players. Journal of Sports Sciences, 27(1), 1-8.

Hill-Haas, S.V., Dawson, B., Impellizzeri, F., \& Coutts, A. (2011). Physiology of small-sided games training in football: A systematic review. Sports Medicine, 41(3), 199-220.

Hodgson, C., Akenhead, R., \& Thomas, K. (2014). Time-motion analysis of acceleration demands of 4v4 small-sided soccer games played on different pitch sizes. Human Movement Science, 33, 25-32.

Hopkins, W.G., Marshall, S.W., Batterham, A.M., \& Hanin, J. (2009). Progressive statistics for studies in sports medicine and exercise science. Medicine and Science in Sports and Exercise, 41(1), 3-12.

Jastrzebski, Z., \& Radziminski, L. (2015). Individual vs general time-motion analysis and physiological response in 4 vs 4 and 5 vs 5 small-sided soccer games. International Journal of Performance Analysis in Sport, 15(1), 397-410.

Katis, A., \& Kellis, E. (2009). Effects of small-sided games on physical conditioning and performance in young soccer players. Journal of Sports Science and Medicine, 8(3), 374-380.

Lehance, C., Binet, J., Bury, T., \& Croisier, J.L. (2009). Muscular strength, functional performances and injury risk in professional and junior elite soccer players. Scandinavian Journal of Medicine and Science in Sports, 19(2), 243-251.

Maio Alves, J., Rebelo, A., Abrantes, C., \& Sampaio, J. (2010). Short-term effects of complex and contrast training in soccer players' vertical jump, sprint, and agility abilities. Journal of Strength and Conditioning Research, 24(4), 936-941.

Ramirez-Campillo, R., Andrade, D.C., \& Izquierdo, M. (2013). Effects of plyometric training volume and training surface on explosive strength. Journal of Strength and Conditioning Research, 27(10), 2714-2722.

Sanchez-Sanchez, J., Hernández, D., Casamichana, D., Martínez-Salazar, C., Ramirez-Campillo, R., \& Sampaio, J. (2017). Heart rate, technical performance and session-RPE in elite youth soccer small-sided games played with wildcard player. Journal of Strength and Conditioning Research, 31(10), 2678-2685.

Stølen, T., Chamari, K., Castagna, C., \& Wisløff, U. (2005). Physiology of soccer. Sports Medicine, 35(6), $501-536$.

Tanaka, H., Monahan, K.D., \& Seals, D.R. (2001). Age-predicted maximal heart rate revisited. Journal of the American College of Cardiology, 37(1), 153-156.

Thomas, J., Silverman, S., \& Nelson, J. (2015). Research methods in physical activity (7 th $^{\text {ed.). }}$. Champaign, IL: Human Kinetics.

Submitted: May 31, 2018

Accepted: February 11, 2020

Published Online First: April 21, 2020
Correspondence to:

Felipe García-Pinillos, Ph.D.

Department of Physical Education, Sport and

Recreation

Universidad de La Frontera, Temuco, Chile

Calle Uruguay, 1980, Temuco, Chile

Phone: Tel+34660062066

E-mail: fegarpi@gmail.com

\section{Acknowledgment}

The authors would like to acknowledge the collaboration of Atlético Menciano and C.D. Egabrense. 